\title{
A novel heterodimeric cytokine consisting of IL-17 and IL-17F regulates inflammatory responses
}

\author{
Seon Hee Chang ${ }^{1}$, Chen Dong ${ }^{1}$ \\ ${ }^{1}$ Department of Immunology, MD Anderson Cancer Center, 7455 Fannin, Unit 906, Houston, TX 77030, USA
}

CD4+ helper T (TH) cells play crucial roles in immune responses. Recently a novel subset of TH cells, termed $\mathrm{TH}_{\mathrm{IL}-17}$, TH17 or inflammatory TH (THi), has been identified as critical mediators of tissue inflammation. These cells produce IL-17 (also called IL-17A) and IL-17F, two most homologous cytokines sharing similar regulations. Here we report that when overexpressed in $293 \mathrm{~T}$ cells, IL-17 and IL-17F form not only homodimers but also heterodimers, which we name as IL-17A/F. Fully differentiated mouse THi cells also naturally secrete IL-17A/F as well as IL-17 and IL-17F homodimeric cytokines. Recombinant IL-17A/F protein exhibits intermediate levels of potency in inducing IL-6 and $\mathrm{KC}$ (CXCL1) as compared to homodimeric cytokines. IL-17A/F regulation of IL-6 and KC expression is dependent on IL-17RA and TRAF6. Thus, IL-17A/F cytokine represents another mechanism whereby T cells regulate inflammatory responses and may serve as a novel target for treating various immune-mediated diseases.

Keywords: inflammation, IL-17, T cells

Cell Research (2007) 17:435-440. doi: 10.1038/cr.2007.35; published online 24 April 2007

\section{Introduction}

IL-17 is the founding member of a new cytokine family that includes 5 additional molecules, IL-17B, IL-17C, IL-17D, IL-17E (also known as IL-25) and IL-17F [1, 2]. IL-17 expression has been previously associated with many inflammatory diseases in humans, such as rheumatoid arthritis, multiple sclerosis, asthma, systemic lupus erythematosus and allograft rejection. In vitro, IL-17 regulates inflammatory responses by inducing the expression of IL-6, Groa, GM-CSF, several chemokines (CCL2, CCL7, CXCL1, and CCL20) and matrix metalloproteinases (MMP3 and MMP13) [1,3]. Moreover, IL-17 and TNF $\alpha$ exhibit synergy in promoting inflammatory gene expression [4]. Deficiencies in IL-17 signaling result in impaired host defense against microbacterial infections [5] and resistance to autoimmune diseases [3, 6-8].

Correspondence: Chen Dong

Tel: +1-713-563-3203; Fax: +1-713-563-0604

E-mail: cdong@mdanderson.org

Received 13 March 2007; revised 25 March 2007; accepted 25 March 2007; published online 24 April 2007
IL-17 binds to and signals through IL-17 receptor A (IL-17RA), a member of the IL-17R family [9]. Recently, it was reported that IL-17RA might form a heterodimer with IL-17RC [10]. IL-17 activates NFאB and MAP kinase pathways, which results in the up-regulation of IL-6 [11, 12]. It was shown that IL-6 induction by IL-17 in mouse embryonic fibroblasts (MEF) is dependent on TRAF6 [12].

Recent efforts to identify the source of IL-17 have revealed a new lineage of Thelper $(\mathrm{TH})$ cells, called $\mathrm{TH}_{\mathrm{IL}-17}$, TH17 or inflammatory TH (THi). Originally found to be regulated by ICOS costimulatory receptor and IL-23 cytokine in vivo [2, 13], IL-6 and TGF $\beta$ have been recently shown to initiate THi differentiation in vitro [14-16], in which IL-23 plays a synergistic role [17].

In addition to IL-17, differentiated THi cells also produce IL-17F upon activation [8]. IL-17F shares the strongest homology with IL-17 and the two genes located in the same chromosome region [2]. A recent study demonstrated coordinated regulation of IL-17 and IL-17F gene transcription during THi differentiation, possibly through chromatin remodeling at this locus [18]. Expression of IL-17F has also been linked with human inflammatory 
diseases, including asthma [19]. Treatment of human airway epithelial cells, vein endothelial cells, and fibroblasts with IL-17F induced the expression of IL-6, IL-8, GRO $\alpha$, ENA-78, transforming growth factor- $\beta$ (TGF- $\beta$ ), MCP-1 (Monocyte chemotactic protein-1, CCL2), G-CSF, granulocyte-macrophage colony-stimulating factor (GM-CSF) and intercellular adhesion molecule-1 (ICAM-1) [19-23]. Although the receptor of IL-17F is yet to be identified, it at least in part may utilize IL-17RA [24].

In the current study, we found that IL-17 and IL-17F, when overexpressed in vitro or derived from mouse THi cells, form not only homodimers but also a heterodimer, IL-17A/F. IL-17A/F, on its own or in synergy with TNF $\alpha$, regulates the expression of IL-6 and $\mathrm{KC}$ (CXCL1, murine homolog of human Gro $\alpha$ ), which is dependent on IL-17RA and TRAF6.

\section{Materials and Methods}

\section{Analysis of 293 cells expressing IL-17 and/or IL-17F \\ IL-17 and IL-17F cDNA were PCR-amplified and cloned into the pcDNA $3.1^{+}$expression vector (Invitrogen) followed by sequencing confirmation. 293T cells were transfected with the pcDNA-IL-17, IL-17F or empty vector by calcium phosphate transfection. After 2 d of culture, supernatants from 293 T cells expressing IL-17, IL-17F or both were collected. Supernatants were resolved on $10 \%$ SDS- PAGE and blotted with a rat anti-IL-17 antibody (BD PharMingen) and a polyclonal rabbit anti-mouse IL-17F antibody we generated (to be described elsewhere). Specific binding was visualized by Su- per Signal substrate (Pierce) and quantified by using an EpiChemi ${ }^{3}$ Darkroom $^{\mathrm{TM}}$ (UVP Bioimaging Systems). For immunoprecipita- tion, $5 \mathrm{ml}$ of supernatant culture was incubated with $1 \mu \mathrm{g}$ of IL-17 antibody or $1 \mu \mathrm{g}$ of affinity-purified IL-17F polyclonal antibody for $4 \mathrm{~h}$ at $4{ }^{\circ} \mathrm{C}$. $20 \mu \mathrm{l}$ of Protein G or Protein A-agarose beads (Sigma) were added to immunoprecipitation mixtures for $2 \mathrm{~h}$ at $4{ }^{\circ} \mathrm{C}$. Beads were washed 3 times with PBS and the bound complexes were eluted with SDS-sample buffer.}

\section{Purification of recombinant cytokines}

Flag and His tags were added to IL-17 or IL-17F cDNAs, respectively, at the carboxyl terminus using PCR and the resulting constructs were evaluated by sequencing and western blot. Homodimers were purified using Flag or His tag affinity columns. To purify heterodimeric protein, supernatants from 293T cells expressing both Flag-tagged IL-17 and His-tagged IL-17F were first applied to His tag affinity column. His-tagged IL-17F was eluted with 250 $\mathrm{mM}$ Imidazole and applied to Flag affinity column. Eluants were dialyzed with PBS and analyzed by western blotting before being used for in vitro stimulation of peritoneal macrophages and mouse embryonic fibroblasts (MEF). Single chain of IL-17A/F heterodimer was generated by using a PCR strategy as described before [25]. His-tagged IL-17F was fused via a (Gly4Ser)3 linker peptide to the carboxy-terminal end of IL-17 and this molecule was designated as IL-17A-L(Linker)-F.

\section{THi preparation}

CD4+ T cells from OT-II mice were differentiated into THi cells as previously described [26]. $5 \mathrm{~d}$ after activation, THi cells were washed twice before restimulation with $500 \mathrm{ng} / \mathrm{ml}$ ionomycin and $50 \mathrm{ng} / \mathrm{ml}$ PMA overnight. $5 \mathrm{ml}$ of supernatants were applied for immunoprecipitation as described above.

Mice

IL-17RA ${ }^{-/}$mice were provided by Amgen (Seattle, Washington) and bred in MD Anderson Cancer Center animal facility. Mouse macrophages were obtained from peritoneal lavage of mice $4 \mathrm{~d}$ after injection with $4 \%$ thioglycollate broth (Difco). After culturing for $3 \mathrm{~h}$, non-adherent cells were removed, and adherent macrophages were used for experiments.

\section{Cell culture}

MEF were derived from C57BL6 using a standard protocol. TRAF6-/- MEF were provided by Dr Tak Mak (Toronto, Canada). MyD88-/- MEF were provided by Dr Ruslan Medzhitov (Yale University, New Haven). For measurement of IL-6 or KC production, $4 \times 10^{4}$ cells were plated onto 24 -well plates. Next day, cells were treated with various cytokines overnight, and the culture supernatants were then analyzed by ELISA.

\section{Results}

\section{IL-17 and IL-17F form a heterodimer}

In order to test the specificity of our polyclonal antiIL-17F antibody in western blotting, we expressed IL-17 and/or IL-17F in 293T cells and immunoblotted the culture supernatants with an anti-IL-17 or anti-IL-17F antibody. As expected, anti-IL-17 only detected secreted proteins from IL-17-expressing cells while anti-IL-17F only detected those from cells expressing IL-17F (Figure 1A). IL-17 migrated as 2 bands between 26 and $37 \mathrm{kDa}$ on a non-reducing gel, indicating homodimer formation and differential glycosylations. On the other hand, IL-17F migrated above $37 \mathrm{kDa}$ as 2 bands. Interestingly, when IL-17 and IL-17F were co-expressed, molecular weights of detected proteins in non-reducing SDS-PAGE gel were different compared with IL-17 or IL-17F expressed alone. Anti-IL-17 detected a higher molecular weight band close to $37 \mathrm{kDa}$ and antiIL-17F revealed a lower molecular weight band at less than $37 \mathrm{kDa}$ (Figure 1A). This observation suggests a possibility of protein complexes distinct from simple IL-17 and IL$17 \mathrm{~F}$ homodimers.

To determine whether IL-17 and IL-17F form a heterodimer, culture supernatants from $293 \mathrm{~T}$ cells overexpressing IL-17 and IL-17F were used for immunoprecipitation. First, anti-IL-17 antibody was used to precipitate any IL17-containing complexes. Precipitates were blotted with anti-IL-17F, and 2 bands were identified (Figure 1B). Similarly, immunoprecipitation of culture supernatants with anti-IL-17F antibody also pulled down IL-17, which confirmed the existence of a heterocomplex. Under reducing conditions, these proteins migrated between 17 and $21 \mathrm{kDa}$ as monomers, consistent with previously reported molecular 
A

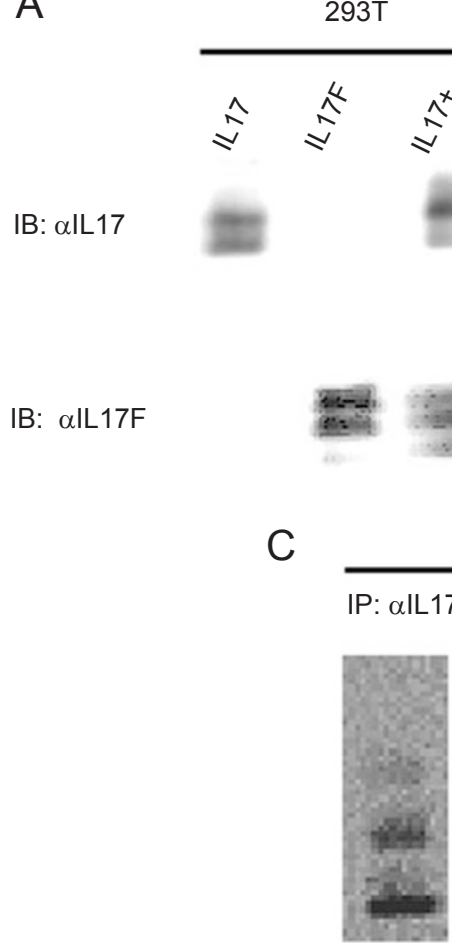

IB: $\quad \alpha$ IL17

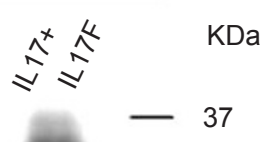

25

- 48
B

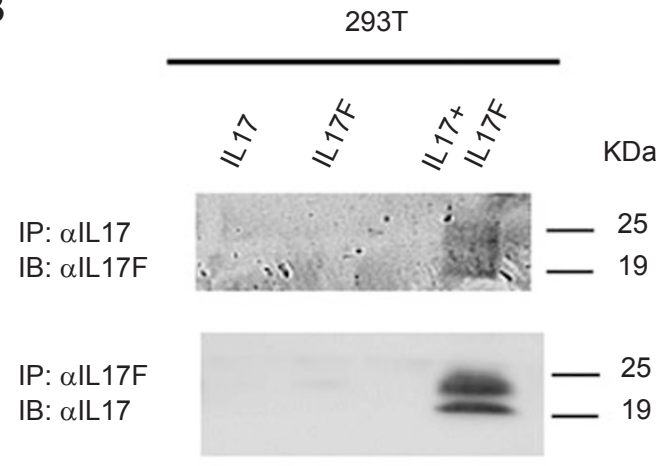

Figure 1 IL-17 forms a heterodimeric complex with IL-17F. (A) Western blot of IL-17 and IL-17F from culture supernatants of $293 \mathrm{~T}$ cells. $48 \mathrm{~h}$ after transfection, culture supernatants of 293 T cells transfected with indicated expression vectors were collected and resolved on non-reducing SDS-PAGE gels and subject to blotting with anti-IL-17 or IL-17F antibody. (B) culture supernatants from 293 T cells expressing IL-17, IL-17F or both were immunoprecipitated (IP) with anti-IL-17 or anti-IL17F antibody and the eluants were applied to reducing SDS-PAGE and immunoblotting (IB) with anti-IL-17 or anti-IL17F antibody. (C) Differentiated THi cells were restimulated with PMA and ionomycin overnight. Supernatants were immunoprecipitated with anti-IL-17 or anti-IL-17F antibody and the eluants were analyzed by immunoblotting.

weight of IL-17 [9] or IL-17F [19]. Thus in addition to homodimers, IL-17 and IL-17F also form a heterodimer when overexpressed in $293 \mathrm{~T}$ cells.

To assess whether THi cells, which co-express IL-17 and IL-17F, also produce heterodimers containing IL-17 and IL-17F, differentiated mouse THi cells were re-stimulated with PMA and Ionomycin overnight and the supernatants were subjected to immunoprecipitation as described above. Anti-IL-17 antibody precipitated 3 forms of IL-17, 2 of which were recognized by anti-IL-17F antibody (Figure 1C). Likewise, anti-IL-17F antibody immunoprecipitated 3 forms of IL-17F and at least one of these forms was recognized by the anti-IL-17 antibody. Interestingly, in these experiments, the species that were immunoprecipitated by either anti-IL-17 or anti-IL-17F but showed reactivity with the other antibody, were found to migrate at the same position in non-reducing gel (Figure 1C). Densitometric quantification of the western blot indicated that approximately
$22.7 \%$ of anti-IL-17 immunoprecipitants were detected by anti-IL-17F antibody and $30.8 \%$ of anti-IL-17F immunoprecipitants were detected by anti-IL-17 antibody.

Thus, IL-17 and IL-17F form a heterodimeric complex not only when they are co-expressed in transfected cells but also as physiologically secreted products from $\mathrm{T}$ cells. We name this novel cytokine molecule IL-17A/F hereafter.

\section{Biological activity of IL-17A/F heterodimer}

To assess the biological activity of the IL-17A/F heterodimer, IL-17 and IL-17F cDNAs were cloned with Flag and His tags at the $\mathrm{C}$-terminus, respectively. The two cytokine homodimers were purified using affinity columns. Purity and identity of the isolated recombinant proteins were confirmed with Commassie blue staining (data not shown) and western blot (Figure 2A). IL-17A/F heterodimer was purified by two-step affinity purification. First, culture supernatants of 293T cells were applied to a His tag affinity column, and 
A

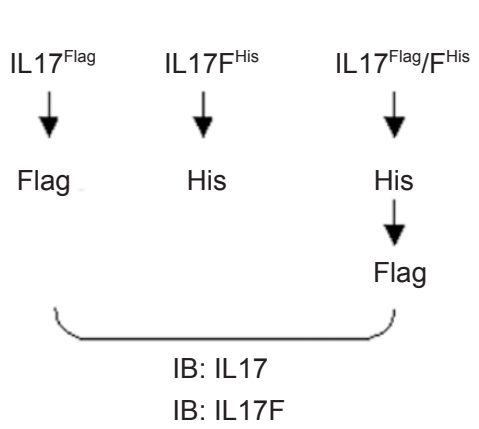

IB: IL17

IL17F

B
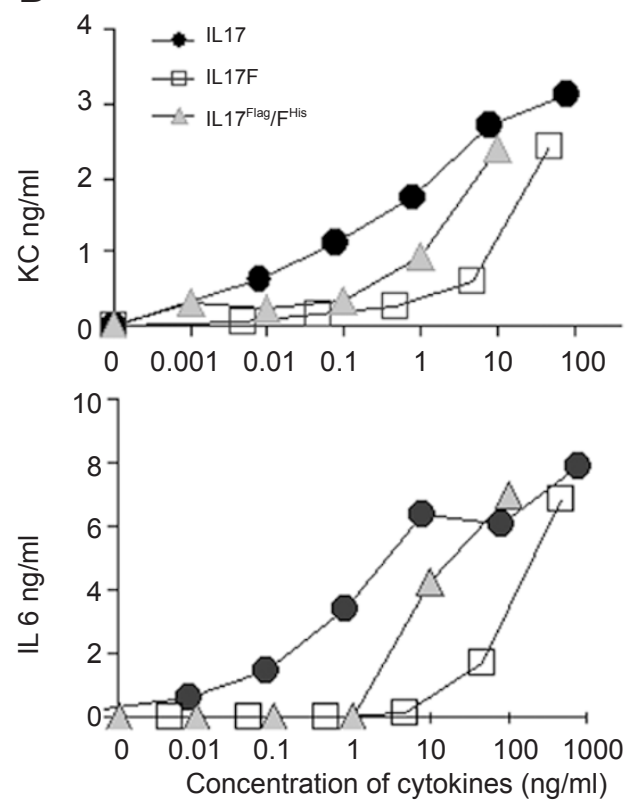

Figure 2 Regulation of inflammatory responses by IL-17A/F. (A) Preparation of recombinant cytokines. Supernatants of 293T cells expressing Flag-tagged IL-17 were applied to a Flag affinity column to purify IL-17 homodimers. His-tagged homodimeric IL-17F was purified using a His-tag affinity column. IL-1 $7^{\text {Flag }} / \mathrm{F}^{\text {His }}$ heterodimer was purified by a His affinity column followed by a Flag affinity column. Purified proteins were run on SDS-PAGE and immunoblotted with anti-IL-17 or anti-IL-17F antibody. (B) MEF were treated with indicated cytokines overnight and culture supernatants were measured for KC and IL-6 levels by ELISA.

then the eluants were added to a Flag tag affinity column. Identity of the IL- $17^{\text {Flag }} / \mathrm{F}^{\mathrm{His}}$ heterodimer was confirmed by western blotting with anti-IL-17 and anti-IL-17F antibodies (Figure 2A).

We next compared the activities of IL-17, IL-17F and IL-17A/F proteins prepared in this manner. IL-17 and IL-17F are known to target fibroblast, epithelial cells and macrophages to produce IL- 6 and KC. When mouse embryonic fibroblasts (MEF) were treated with different doses of recombinant cytokines, IL-17 was most potent in inducing IL-6 and KC (Figure 2B). IL-17A/F heterodimer has intermediate activity between IL-17 and IL-17F. However, at $>100 \mathrm{ng} / \mathrm{ml}$ concentrations, the ability of these cytokines to induce IL-6 or KC expression was indistinguishable.

We also generated a single chain of IL-17A/F protein, in which IL-17 was fused with IL-17F via a linker. The resulting protein was purified via His tag affinity column and named as IL-17A-L-F. Similar to IL-1 $7^{\text {Flag }} / \mathrm{F}^{\mathrm{His}}$, recombinant IL-17A-L-F was able to synergize with TNF $\alpha$ to induce the $\mathrm{KC}$ production by wild-type macrophages (Figure $3 \mathrm{~A}$ ).

Our results thus demonstrate that recombinant IL-17A/F proteins are biologically active and regulate pro-inflammatory responses.
Signaling requirements of the IL-17A/F heterodimer

IL-17 mediates inflammatory responses dependent on IL-17RA and TRAF6. To understand the receptor usage of IL-17A/F, peritoneal macrophages from wild-type and IL17RA-deficient mice were isolated. Upon treatment with IL-17, IL-17 Flag $^{\text {F }}$ His or IL-17A-L-F, there was no induction of $\mathrm{KC}$ or IL-6 in wild-type or knockout cells. However, similar to IL-17, recombinant IL-17A/F proteins synergized with $\mathrm{TNF} \alpha$ to increase $\mathrm{KC}$ expression (Figure 3A). Increase of KC expression by IL-17, IL-17 $7^{\text {Flag }} / \mathrm{F}^{\mathrm{His}}$ or IL-17A-L-F was impaired in the absence of IL-17RA (Figure 3A), indicating IL-17A/F also utilizes IL-17RA as its receptor.

We also tested the involvement of TRAF6, a signaling molecule that associates with IL-17RA and Act1 adaptor protein [27]. While KC or IL-6 induction by IL-17 $7^{\text {Flag }} / \mathrm{F}^{\mathrm{His}}$ or IL-17A-L-F was preserved in MyD88-/- MEF, their production was abolished in TRAF6 KO MEF (Figure 3B).

\section{Discussion}

While THi cells secret IL-17 and IL-17F upon activation, we found that an additional cytokine, consisting of heterodimeric IL-17A/F, is also produced by THi cells. 
A

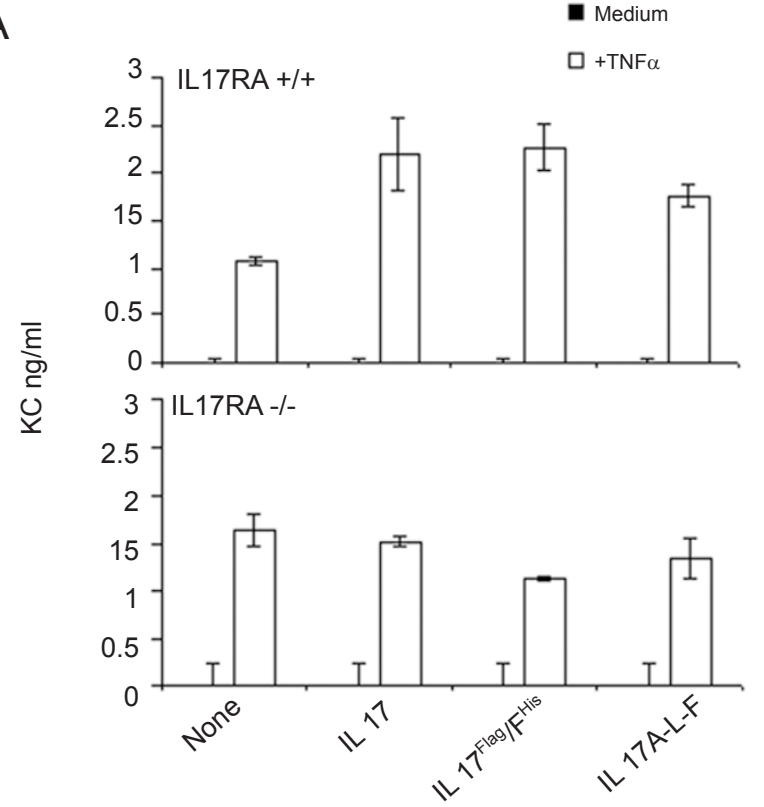

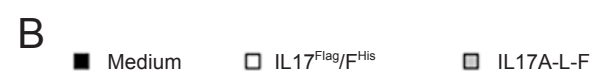
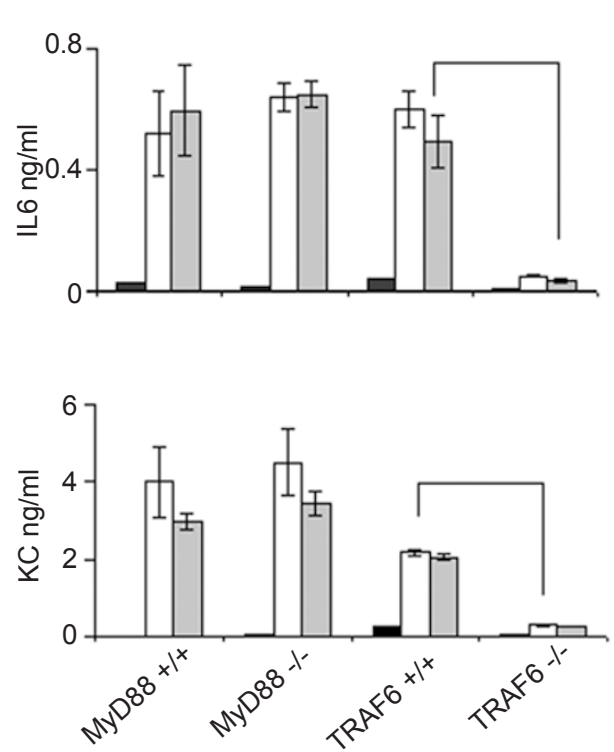

Figure 3 IL-17A/F signals through IL-17RA and TRAF6. (A) WT and IL-17RA KO peritoneal macrophages were treated with IL-17, IL-1 $7^{\mathrm{Flag}} / \mathrm{F}^{\mathrm{His}}$ and IL-17A-L-F in the absence or presence of TNF $\alpha .24 \mathrm{~h}$ after treatment, KC was measured from culture supernatants by ELISA. Asterisk indicates statistically significant differences as determined by two-tailed Student's test $(P<0.05)$. (B) Indicated MEF were treated with IL-1 $7^{\text {Flag }} / \mathrm{F}^{\mathrm{His}}$ and IL-17A-L-F overnight and the culture supernatants were measured for KC and IL-6 levels (Asterisk indicates $P<0.05$ ).

Recombinant IL-17A/F functions very similarly as its homodimeric counterparts. It targets fibroblasts or macrophages to induce pro-inflammatory molecules such as IL-6 and KC. Our study thus suggests that IL-17 and IL-17F promote inflammation in the forms of homodimers and heterodimers.

Identification of the IL-17A/F heterodimer may reveal further complexity in receptor usage by IL-17 cytokines in promoting inflammatory responses. While it is generally accepted that IL-17 utilizes IL-17RA for its signaling and IL-17RA forms complex with IL-17RC, the receptor for IL-17F is not clear. It has been suggested that IL-17F may use the same receptor, IL-17RA, although with different affinity since IL-17F did not bind to IL-17RA in vitro. Our study revealed that IL-17A/F also requires IL-17RA for its signaling. Although it is likely that all of these cytokine molecules act in concert to create a pro-inflammatory environment, one cannot exclude the possibility that these molecules may compete for the binding to IL-17RA or other receptors in vivo.

So far, the IL-17A/F heterodimer did not exhibit any unique biological property compared with its homodimeric counterparts. However, the role of IL-17A/F in vivo should be examined more thoroughly, for instance, by overexpres- sion in mice. Also, development of blocking antibodies or antagonistic peptides that react with the IL-17A/F heterodimer in addition to respective homodimers would be more appropriate to inhibit inflammatory responses driven by the IL-17 family.

THi cells now are recognized as a distinct lineage of CD4 helper $\mathrm{T}$ cells that produce heterogeneous combinations of inflammatory molecules. It was demonstrated recently that IL-22, a pro-inflammatory molecule, is also derived from THi cells and synergizes with IL-17 and IL-17F to induce the production of antimicrobial peptides [26, 28]. Therefore, identification of the IL-17A/F heterodimer, emphasizes the inflammatory role of THi cells in vivo. Future studies should be considered to determine the effect of the IL-17A/F heterodimer in cooperation with its homodimeric counterparts and other pro-inflammatory cytokines on development and progression of human diseases.

In conclusion, we found that THi cells upon stimulation produce not only IL-17 and IL-17F homodimers but also the IL-17A/F heterodimer. Similar to homodimers, IL-17A/F activates fibroblasts and macrophages to produce proinflammatory mediators such as KC and IL-6. IL-17A/F signals through IL-17RA and TRAF6. These results reveal a novel mechanism whereby $\mathrm{T}$ cells regulate inflammatory 
responses. Considering the similar actions of IL-17, IL-17F and IL-17A/F, it may be beneficial to target all of them in many inflammatory diseases that now have been found to be mediated by THi cells.

\section{Acknowledgments}

We thank Dr Ruslan Medzhitov (Yale University) for MyD88-deficient MEF, Dr Tak Mak (Toronto, Canada) for TRAF6-deficient MEF, Amgen (Seattle) for IL-17RA KO mice and the entire Dong Lab for help and discussion. This work was in part supported by National Institutes of Health (to Dong C). Chang SH receives a post-doctoral fellowship from the Arthritis foundation and Dong $\mathrm{C}$ is a Cancer Research Institute Investigator, an American Lung Association Career Investigator and a MD Anderson Cancer Center Trust Fellow.

\section{References}

1 Moseley TA, Haudenschild DR, Rose L, Reddi AH. Interleukin17 family and IL-17 receptors. Cytokine Growth Factor Rev 2003; 14:155-174.

2 Dong C. Diversification of T-helper-cell lineages: finding the family root of IL-17-producing cells. Nat Rev Immunol. 2006; 6:329-333.

3 Park H, Li Z, Yang XO, et al. A distinct lineage of CD4 T cells regulates tissue inflammation by producing interleukin 17 . Nat Immunol 2005; 6:1133-1141.

4 Ruddy MJ, Wong GC, Liu XK, et al. Functional cooperation between interleukin-17 and tumor necrosis factor-alpha is mediated by CCAAT/enhancer-binding protein family members. J Biol Chem 2004; 279:2559-2567.

5 Ye P, Rodriguez FH, Kanaly S, et al. Requirement of interleukin 17 receptor signaling for lung $\mathrm{CXC}$ chemokine and granulocyte colony-stimulating factor expression, neutrophil recruitment, and host defense. J Exp Med 2001; 194:519-527.

6 Nakae S, Nambu A, Sudo K, Iwakura Y. Suppression of immune induction of collagen-induced arthritis in IL-17-deficient mice. J Immunol 2003; 171:6173-6177.

7 Nurieva RI, Mai XM, Forbush K, Bevan MJ, Dong C. B7h is required for $\mathrm{T}$ cell activation, differentiation, and effector function. Proc Natl Acad Sci USA 2003; 100:14163-14168.

8 Langrish CL, Chen Y, Blumenschein WM, et al. IL-23 drives a pathogenic $\mathrm{T}$ cell population that induces autoimmune inflammation. J Exp Med 2005; 201:233-240.

9 Yao Z, Fanslow WC, Seldin MF, et al. Herpesvirus Saimiri encodes a new cytokine, IL-17, which binds to a novel cytokine receptor. Immunity 1995; 3:811-821.

10 Toy D, Kugler D, Wolfson M, et al. Cutting edge: interleukin 17 signals through a heteromeric receptor complex. J Immunol 2006; 177:36-39.

11 Shalom-Barak T, Quach J, Lotz M. Interleukin-17-induced gene expression in articular chondrocytes is associated with activation of mitogen-activated protein kinases and NF-kappaB. J Biol Chem 1998; 273:27467-27473.

12 Schwandner R, Yamaguchi K, Cao Z. Requirement of tumor necrosis factor receptor-associated factor (TRAF) 6 in interleukin 17 signal transduction. J Exp Med 2000;191:1233-1240.

13 Dong C, Nurieva RI. Regulation of immune and autoimmune responses by ICOS. J Autoimmun 2003; 21:255-260.

14 Bettelli E, Carrier Y, Gao W, et al. Reciprocal developmental pathways for the generation of pathogenic effector TH17 and regulatory T cells. Nature 2006; 441:235-238.

15 Mangan PR, Harrington LE, O'Quinn DB, et al. Transforming growth factor-beta induces development of the $\mathrm{T}(\mathrm{H}) 17$ lineage. Nature 2006; 441:231-234.

16 Veldhoen M, Hocking RJ, Atkins CJ, Locksley RM, Stockinger B. TGFbeta in the context of an inflammatory cytokine milieu supports de novo differentiation of IL-17-producing $\mathrm{T}$ cells. Immunity 2006; 24:179-189.

17 Yang XO, Panopoulos AD, Nurieva R, et al. STAT3 regulates cytokine-mediated generation of inflammatory helper T cells. J Biol Chem 2007; 282:9358-9363.

18 Akimzhanov AM, Yang XO, Dong C. Chromatin remodeling at IL17-IL-17F cytokine gene locus during inflammatory helper T cell differentiation. J Biol Chem 2007; 282:5969-5972.

19 Kawaguchi M, Onuchic LF, Li XD, et al. Identification of a novel cytokine, ML-1, and its expression in subjects with asthma. J Immunol 2001; 167:4430-4435.

20 Hymowitz SG, Filvaroff EH, Yin JP, et al. IL-17s adopt a cystine knot fold: structure and activity of a novel cytokine, IL-17F, and implications for receptor binding. Embo J 2001; 20:53325341 .

21 Starnes T, Robertson MJ, Sledge G, et al. Cutting edge: IL-17F, a novel cytokine selectively expressed in activated $\mathrm{T}$ cells and monocytes, regulates angiogenesis and endothelial cell cytokine production. J Immunol 2001; 167:4137-4140.

22 Kawaguchi M, Kokubu F, Matsukura S, et al. Induction of C-X$\mathrm{C}$ chemokines, growth-related oncogene alpha expression, and epithelial cell-derived neutrophil-activating protein- 78 by ML-1 (interleukin-17F) involves activation of Rafl-mitogen-activated protein kinase kinase-extracellular signal-regulated kinase 1/2 pathway. J Pharmacol Exp Ther 2003; 307:1213-1220.

23 Kawaguchi M, Kokubu F, Odaka M, et al. Induction of granulocyte-macrophage colony-stimulating factor by a new cytokine, ML-1 (IL-17F), via Raf I-MEK-ERK pathway. J Allergy Clin Immunol 2004;114:444-450.

24 McAllister F, Henry A, Kreindler JL, et al. Role of IL-17A, IL-17F, and the IL-17 receptor in regulating growth-related oncogene-alpha and granulocyte colony-stimulating factor in bronchial epithelium: implications for airway inflammation in cystic fibrosis. J Immunol 2005;175:404-412.

25 Degen WG, van Daal N, van Zuilekom HI, Burnside J, Schijns VE. Identification and molecular cloning of functional chicken IL-12. J Immunol 2004; 172:4371-4380.

26 Chung Y, Yang X, Chang SH, Ma L, Tian Q, Dong C. Expression and regulation of IL-22 in the IL-17-producing CD4+ T lymphocytes. Cell Res 2006; 16:902-907.

27 Chang SH, Park H, Dong C. Act1 adaptor protein is an immediate and essential signaling component of interleukin-17 receptor. J Biol Chem 2006; 281:35603-35607.

28 Liang SC, Tan XY, Luxenberg DP, et al. Interleukin (IL)-22 and IL-17 are coexpressed by Th17 cells and cooperatively enhance expression of antimicrobial peptides. J Exp Med 2006; 203:22712279. 